\title{
SGLT2 Inhibitors and the Clinical Implications of Associated Weight Loss in Type 2 Diabetes: A Narrative Review
}

\author{
Andrej Janež $\cdot$ Paola Fioretto
}

Received: April 15, 2021 / Accepted: June 3, 2021 / Published online: July 9, 2021

(C) The Author(s) 2021

\begin{abstract}
Introduction: The obesity epidemic is closely linked to the rising prevalence of type 2 diabetes (T2D). Body weight reduction remains an important challenge in patients with $\mathrm{T} 2 \mathrm{D}$, as it requires changing their overall metabolic control. Of all glucose-lowering therapies, only sodium-glucose cotransporter 2 inhibitors (SGLT2is) and glucagon-like peptide 1 receptor agonists (GLP-1 RAs) consistently result in weight improvement. Moreover, the same two classes have important cardiovascular and renal benefits. We summarize the key available information related to the weight loss effect of SGLT2is in T2D, focusing on the unexploited potential of these drugs.
\end{abstract}

\footnotetext{
A. Janež $(\bowtie)$

Department of Endocrinology, Diabetes and Metabolic Diseases, University Medical Centre Ljubljana, Zaloška Cesta 7, 1000 Ljubljana, Slovenia e-mail: andrej.janez@kclj.si

P. Fioretto

Department of Medicine, University of Padua, Padua, Italy

P. Fioretto

Unit of Medical Clinic 3, Hospital of Padua, Padua, Italy
}

Methods: Data on weight change with SGLT2is in patients with T2D were extracted from published cardiovascular outcomes trials (CVOTs). A discussion on patient perspectives about weight change is based on key preclinical and clinical trials, meta-analyses, and reviews and is supplemented by the authors' clinical judgment and research experience in the field.

Results: SGLT2is have a unique mode of action resulting in caloric loss through glycosuria. The anticipated weight loss with SGLT2is is not reflected in clinical trial results. There is a discrepancy between the magnitude of improvement in glycemic control and the weight loss, cardiovascular, and renal benefits obtained in large clinical trials.

Conclusion: The relationships between the magnitude of weight loss, improvement in glycemic control, and cardiorenal benefits with SGLT2i are still unclear. Potential mechanisms other than simple glycemic efficacy should be revealed and explained. Better weight control may be achieved if adequately intensive lifestyle changes are implemented and monitored in the T2D population treated with SGLT2is.

Keywords: CVOT; SGLT2 inhibitors; Type 2 diabetes; Weight loss 


\section{Key Summary Points}

Why carry out this review?

Obesity is closely linked to type 2 diabetes, and it is often difficult to achieve and maintain the control of these conditions.

SGLT2 inhibitors are among a few therapeutic options with clear benefits on weight management.

Accumulating evidence also demonstrates the important cardiorenal risk reduction in this patient population.

\section{What was learned from this review?}

The mechanisms by which SGLT2 inhibition leads to cardiorenal risk reduction are not fully elucidate, but reduction is an important component.

A structured approach to enhance SGLT2 inhibitor effects on weight may be implemented in clinical practice with the potential to enhance healthy behaviors to yield optimal outcomes in patients with type 2 diabetes.

\section{DIGITAL FEATURES}

This article is published with digital features, including a summary slide, to facilitate understanding of the article. To view digital features for this article go to https://doi.org/10.6084/ m9.figshare.14718531.

\section{INTRODUCTION}

Type 2 diabetes (T2D) is a chronic disease with a major impact on patients' lives and health care systems. Its prevalence ranges from $7 \%$ to $25 \%$ [1] in European Union countries, and it is closely associated with overweight, obesity, and physical inactivity. Current estimates project an increase in T2D incidence of up to $20 \%$ in
Europe in the next 20 years $[1,2]$. In recent years, a new term has emerged, diabesity, reflecting the lack of weight control in patients with T2D [3] and the urgent need to find efficient strategies to address both diabetes and obesity.

Lifestyle changes promoting a reduction in caloric intake and an increase in energy expenditure represent the first-line action [4]. Patients with diabetes are educated on how to integrate a balanced, calorie-restricted diet and at least moderate physical activity in their routine. However, the implementation of daily healthy behavior is out of the strict control of the medical team, relying mostly on patients' understanding, motivation, and commitment to reaching realistic goals. Achievement and maintenance of normal weight is one of the most important treatment objectives for patients with T2D. Current therapeutic guidelines for T2D favor the early use of pharmacotherapies with demonstrated cardiovascular $(\mathrm{CV})$ and renal benefits and a positive impact on weight control, irrespective of HbA1c value [4].

The development of sodium-glucose cotransporter 2 (SGLT2) inhibitors (SGLT2is) represents a major turning point in the management of diabetes owing to their unique mechanism of action and proven cardiovascular and renal benefits. One of the first reported additional benefits of SGLT2is was the weight loss effect, a result of caloric excretion and fat mass reduction.

\section{Mechanism of Action and Effect on Energy Balance}

Glycosuria is one of the key characteristics of poorly controlled diabetes, along with hyperglycemia and reduced insulin secretion and/or glucose utilization. Kidneys play a key role in maintaining glucose homeostasis, participating in steps along the whole metabolic chain of glucose, including its production (gluconeogenesis), utilization, filtration, reabsorption, and excretion.

SGLT2 is a high-capacity, low-affinity glucose transporter responsible for $90 \%$ of glucose reabsorption [5]. SGLT2 transporters are located 
in kidneys, mainly in the S1 and S2 segments of the proximal convoluted tubules [6]. The rest of the glucose is further reabsorbed in the S3 segment by SGLT1, a low-capacity, high-affinity transporter. Tubular reabsorption reaches its maximum capacity at approximately $375 \mathrm{mg}$ glucose/min, with a corresponding plasma glucose level of $200 \mathrm{mg} / \mathrm{dL}$. Glycosuria occurs when this threshold is exceeded. If the hyperglycemic status is persistent, enhanced SGLT1/2 expression leads to paradoxically higher rates of glucose reabsorption [7].

The development of SGLT2is represents a major paradigm shift in the treatment of diabetes, turning a "defect" into a "mode of action". Four SGLT2is are currently approved in Europe for T2D treatment in combination with diet, exercise, and lifestyle changes (canagliflozin, dapagliflozin, empagliflozin, and ertugliflozin). They are not specifically approved by regulatory agencies for body weight reduction or treatment of obesity.

\section{SGLT2 Inhibition: Effect on Body Weight}

At therapeutic doses, urinary glucose excretion (UGE) is approximately 70-90 g/day, equivalent to $300 \mathrm{kcal} / \mathrm{day}$, with additional diuresis of $400 \mathrm{~mL} /$ day $[8,9]$. On the basis of the measured number of calories lost per day due to UGE, the expected weight loss would be approximately $10 \mathrm{~kg} /$ year [7]. The actual loss in body weight is much less, as shown in clinical trials and in clinical practice [10]. The relationship between SGLT2 inhibition and the caloric effect turned out to be much more complex and not a simple linear function, as initially thought [11]. Clinical trials and observational data show that weight reduction is lower than expected (approximately $2-5 \mathrm{~kg}$ ), with variations according to baseline weight and concomitant medication. The body weight reduction during treatment with SGLT2is has been considered modest, though it provides large cardiovascular benefits. The mechanisms of weight loss and their influence on cardiorenal effects seem complex and are not fully elucidated.

This short review explores how the weight loss achieved with SGLT2is might influence multiple outcomes in T2D. Our aim is to briefly review the weight-related results reported in the context of cardiovascular outcomes trials (CVOTs) and discuss the possible implications for clinical practice.

\section{METHODS}

This is a narrative review based on a literature search in the PubMed database up to February 26, 2021. The search algorithm covered SGLT2 inhibitors or inhibition, canagliflozin, dapagliflozin, empagliflozin, ertugliflozin in connection with weight loss, weight change, metabolic change, adipose tissue, body composition, and CVOTs. Documentation was based on information from full-text publications, including preclinical and clinical trials, metaanalyses, and reviews on this topic. The search strategy was limited to English-language articles. Case reports on weight change with SGLT2is were not included. CVOTs in patients with T2D only were selected. Data extraction was conducted by one reviewer and revised by one coauthor. The selected references were individually searched to identify more information on the topic. The discussion is supplemented by the authors' research experience and clinical judgment.

\section{Compliance with Ethics Guidelines}

This article is based on previously conducted studies and does not contain any new studies with human participants or animals performed by any of the authors.

\section{RESULTS}

\section{Difference Between Estimated Energy Loss and Actual Weight Reduction}

Several years ago, Rajeev et al. [8] explored the discrepancy between the anticipated effect of SGLT2is and actual weight loss reported in clinical trials. They described several adaptive changes, such as compensatory hyperphagia, 
increased gluconeogenesis, and a shift toward fatty acid utilization as a metabolic substrate.

Evidence to support compensatory hyperphagia is limited. In rodent models, lack of SGLT2 expression or chronic administration of dapagliflozin or canagliflozin led to weight reduction and increased caloric intake, considered to be compensatory to energy loss [12-14]. Similar predictions come from mathematical models with canagliflozin [15] or empagliflozin [16], showing several-fold increases in calorie intake compared to the adaptation in energy expenditure. However, this theory was not supported by a 12 -week randomized controlled trial with dapagliflozin vs. placebo, where weight loss obtained with SGLT2 inhibition was not associated with a significant increase in food intake $(p=0.659)$ [17]. Another clinical trial in patients with T2D [18] showed similar results, with no significant association of dapagliflozin with compensatory carbohydrate intake.

Another possible explanation for the limited body weight decrease is an increase in glucagon effects, with promotion of renal and hepatic gluconeogenesis and a consequent increase in endogenous glucose production. However, the role of SGLT2 inhibition in endocrine regulation remains unclear.

Compensatory metabolic and endocrine processes do not totally counteract the weight loss effect. Clinical trials with SGLT2is have shown a biphasic pattern of weight loss: an initial large effect, probably resulting from enhanced fluid elimination, followed by a slow increase, with mean weights lower than baseline at any assessment $[19,20]$. The gradual effect is maintained for up to 4 weeks with dapagliflozin [21] and has been explained by the reduction in visceral and subcutaneous fat mass [21-23].

\section{A Closer Look into Weight Changes Reported in CVOTs}

Developed for T2D treatment and currently used as antihyperglycemic drugs, SGLT2is have proven cardiovascular and renal benefits in large CVOTs. Since the primary and key secondary objectives have focused on the reduction of $\mathrm{CV}$ and renal events, weight changes were only briefly reported in the primary manuscripts (Table 1) [24-28].

\section{CANVAS Program}

A post hoc analysis [29] showed similar cardiorenal results between different baseline body mass index (BMI) groups. The authors acknowledged a substantial variation in weight change over time. The weight decrease with canagliflozin vs. placebo was larger at the 12-month follow-up $(-2.77 \%$; $95 \%$ CI -2.95 , - 2.59) than at the 3-month follow-up ($1.72 \%$; 95\% CI - 1.83, - 1.62). The most plausible explanation resides in the time-additive effects of the dual mechanisms of weight loss with SGLT2is. A dose-dependent effect was observed at both time points. Early effects were positively associated with coadministration of insulin or glucagon-like peptide 1 receptor agonists (GLP-1 RA), as well as the absence of a history of heart failure or arterial hypertension (all $p$ for trend $<0.05$ ); however, a possible explanation was not apparent. A contradictory result was the larger weight reduction at 3 months in people with lower initial HbA1c. Nevertheless, the baseline weight did not influence the prevention of $\mathrm{CV}$ events by canagliflozin.

\section{CREDENCE Trial}

The CREDENCE trial was designed to primarily assess the renal outcomes with canagliflozin $100 \mathrm{mg}$ /day vs. placebo in patients with T2D and reduced kidney function [25]. The average change in weight with canagliflozin vs. placebo (- $0.8 \mathrm{~kg}$; 95\% CI $0.69,0.92)$ [25] was maintained irrespective of the initial glomerular filtration rate [30].

\section{DECLARE-TIMI 58 Trial}

The DECLARE-TIMI 58 trial outcomes (composite risk of $\mathrm{CV}$ disease and hospitalization for heart failure and its components, including CV and renal events) were analyzed according to 
Table 1 Summary of weight change results with SGLT2is in CVOTs including patients with T2D only

\begin{tabular}{|c|c|c|c|c|c|}
\hline $\begin{array}{l}\text { CVOT name } \\
\text { and SGLT2i }\end{array}$ & CVOT study population & $\begin{array}{l}\text { Mean } \\
\text { follow-up } \\
\text { (SD), years }\end{array}$ & $\begin{array}{l}\text { Baseline } \\
\text { weight } \\
\text { (SD), kg }\end{array}$ & $\begin{array}{l}\text { Weight } \\
\text { reduction } \\
\text { (SD) with the } \\
\text { SGLT2i, kg }\end{array}$ & $\begin{array}{l}\text { Risk reduction for the } \\
\text { primary objective }\end{array}$ \\
\hline $\begin{array}{l}\text { CANVAS } \\
\text { [24]: } \\
\text { canagliflozin }\end{array}$ & $\begin{array}{l}\text { T2D and either symptomatic } \\
\text { ASCVD or multiple CV } \\
\text { risk factors } \\
N=10,142 \\
\text { Mean age }(\mathrm{BL})=63.3 \text { years } \\
\text { Mean HbAlc }(\mathrm{BL})=8.2 \%\end{array}$ & $3.6(2.0)$ & $\begin{array}{l}90.2 \\
(\mathrm{NR})\end{array}$ & $-1.6(2.42)$ & $\begin{array}{l}\text { 3P-MACE } \\
\text { HR }=0.86 ; 95 \% \text { CI } \\
0.75-0.97 \\
P<0.001 \text { (non- } \\
\text { inferiority) } \\
P=0.02 \text { (superiority) vs. } \\
\text { placebo }\end{array}$ \\
\hline $\begin{array}{l}\text { CREDENCE } \\
\text { [25]: } \\
\text { canagliflozin }\end{array}$ & $\begin{array}{l}\mathrm{T} 2 \mathrm{D} \text { and } \mathrm{CKD} \text { and } \\
\quad \text { albuminuria } \\
N=4401 \\
\text { Mean age }(\mathrm{BL})=63 \text { years } \\
\text { Mean } \mathrm{HbAlc}(\mathrm{BL})=8.3 \%\end{array}$ & $\begin{array}{l}2.6 \\
\quad(0.02-4.53)\end{array}$ & $\begin{array}{l}87.1 \\
\quad(\mathrm{NR})\end{array}$ & $-0.8(1.95)$ & $\begin{array}{l}\text { Composite of } \\
\text { ESRD/doubling of } \\
\text { serum creatinine vs. } \\
\text { baseline/CV or renal } \\
\text { death } \\
\mathrm{HR}=0.70 ; 95 \% \mathrm{CI} \\
0.59-0.82 \\
P=0.00001 \text { vs. placebo }\end{array}$ \\
\hline $\begin{array}{l}\text { DECLARE- } \\
\text { TIMI } 58 \\
\text { [26]: } \\
\text { dapagliflozin }\end{array}$ & $\begin{array}{l}\text { T2D and either symptomatic } \\
\text { atherosclerotic CV disease } \\
\text { or multiple CV risk factors } \\
N=17,160 \\
\text { Mean age }(\mathrm{BL})=64.0 \text { years } \\
\text { Mean HbAlc }(\mathrm{BL})=8.3 \%\end{array}$ & $4.2(3.9-4.4)$ & $\begin{array}{l}91.0 \\
(\mathrm{NR})\end{array}$ & $-1.8(5.0)$ & $\begin{array}{l}\text { 3P-MACE } \\
\mathrm{HR}=0.93 ; 95 \% \mathrm{CI} \\
0.84-1.03 \\
P=0.17 \text { vs. placebo } \\
\mathrm{CV} \text { death } / \mathrm{hHF} \\
\mathrm{HR}=0.83 ; 95 \% \mathrm{CI} \\
0.73-0.95 \\
P=0.005 \text { vs. placebo }\end{array}$ \\
\hline $\begin{array}{l}\text { EMPA-REG } \\
\text { [27]: } \\
\text { empagliflozin }\end{array}$ & $\begin{array}{l}\text { T2D and established ASCVD } \\
N=7020 \\
\text { Mean age }(\mathrm{BL})=63.1 \text { years } \\
\text { Mean HbAlc }(\mathrm{BL})=8.1 \%\end{array}$ & $3(2.2-3.6)$ & $\begin{array}{l}86.3 \\
\quad(19.0)\end{array}$ & $-2.00(\mathrm{NR})$ & $\begin{array}{l}\text { 3P-MACE } \\
\mathrm{HR}=0.86 ; 95 \% \mathrm{CI} \\
0.74-0.99 \\
P=0.04 \text { (superiority) }\end{array}$ \\
\hline
\end{tabular}


Table 1 continued

\begin{tabular}{|c|c|c|c|c|c|}
\hline $\begin{array}{l}\text { CVOT name } \\
\text { and SGLT2i }\end{array}$ & CVOT study population & $\begin{array}{l}\text { Mean } \\
\text { follow-up } \\
\text { (SD), years }\end{array}$ & $\begin{array}{l}\text { Baseline } \\
\text { weight } \\
\text { (SD), kg }\end{array}$ & $\begin{array}{l}\text { Weight } \\
\text { reduction } \\
\text { (SD) with the } \\
\text { SGLT2i, kg }\end{array}$ & $\begin{array}{l}\text { Risk reduction for the } \\
\text { primary objective }\end{array}$ \\
\hline $\begin{array}{l}\text { VERTIS CV } \\
\text { [28]: } \\
\text { ertugliflozin }\end{array}$ & $\begin{array}{l}\text { Established ASCVD involving } \\
\text { the coronary, } \\
\text { cerebrovascular, and/or } \\
\text { peripheral arterial system } \\
N=8246 \\
\text { Mean age }(\mathrm{BL})=64.4 \text { years } \\
\text { Mean HbAlc }(\mathrm{BL})=8.2 \%\end{array}$ & 3.5 & $\begin{array}{l}\text { NR } \\
\text { Baseline } \\
\text { BMI: } \\
31.9 \\
(5.4) \\
\mathrm{kg} / \mathrm{m}^{2}\end{array}$ & $\begin{array}{l}\text { At } 1 \text { year: } \\
-2.4(3.9) \mathrm{kg} \\
\text { with } 5 \mathrm{mg} \\
-2.8(4.0) \mathrm{kg} \\
\text { with } 15 \mathrm{mg}\end{array}$ & $\begin{array}{l}\text { 3P-MACE } \\
\text { HR }=0.97 ; 95 \% \mathrm{CI} \\
0.85-1.11 \\
P<0.001 \text { vs. placebo }\end{array}$ \\
\hline
\end{tabular}

3P MACE 3-point major adverse cardiovascular events (cardiovascular death, nonfatal myocardial infarction or nonfatal stroke, $A S C V D$ atherosclerotic cardiovascular disease, $B L$ baseline, $B M I$ body mass index, $C K D$ chronic kidney disease, $C I$ confidence interval, $C V$ cardiovascular, $C V O T$ cardiovascular outcome trial, ESRD end-stage renal disease, HbAIc glycated hemoglobin, $H R$ hazard ratio, $N R$ not reported, $T 2 D$ type 2 diabetes, $S D$ standard deviation, $S G L T 2 i$ sodium-glucose cotransporter 2 inhibitor

baseline BMI categories (normal to very obese) [31]. A similar reduction in body weight (approximately $2 \%$ vs. placebo) and similar relative risk for the composite event were observed across the BMI categories.

\section{EMPA-REG Outcomes Trial}

A post hoc analysis of EMPA-REG OUTCOMES data [32] evaluating the early benefits showed a significant weight reduction with empagliflozin vs. placebo $(p<0.0001)$ at 12 weeks, 6 months, and 1 year. The clinical and metabolic benefits were apparent in patients with T2D with or without heart failure at baseline and were considered largely independent of HbA1c reduction.

\section{VERTIS CV Trial}

The weight change observed with ertugliflozin $5 \mathrm{mg}$ or $15 \mathrm{mg}$ in the VERTIS CV trial [28] was slightly lower than that previously reported. The results from another trial, although not a CVOT, showed that weight loss at 26 weeks was maintained for a longer period of time, up to 104 weeks [33]. No specific results from VERTIS $\mathrm{CV}$ on weight change with ertugliflozin by baseline BMI or HbA1c group have been published, but another clinical trial with ertugliflozin in overweight and obese patients concluded that achievement of glycemic control at 26 weeks (HbA1c reduction) was similar irrespective of baseline BMI, and so was the percentage of weight loss (approximately 3-4\%) [34].

The impressive cardiorenal outcomes are only partially explained by the SGLT2is' effects on the heart, kidneys, blood vessels, and the whole body [35]. Potential mechanisms include structural (reduction of ventricular mass and wall stress, improvement of fibrosis markers and endothelial function), dynamic (direct inhibition of myocardial $\mathrm{Na}^{+} / \mathrm{H}^{+}$pump, reduction of blood pressure, and improvement of the ventricular loading conditions and tubuloglomerular feedback), and energetic changes (improvement of cardiac metabolism).

In this context, the associated weight loss has been investigated as a potential distinctive mediator of $\mathrm{CV}$ risk reduction in diabetes. A 
recent systematic review and meta-analysis [36] of large CVOTs with different glucose-lowering drugs showed a significant relative reduction in the risk of heart failure of $5.9 \%(3.9-8.0 \%)$ for every difference of $1 \mathrm{~kg}$ between treatment groups $(p<0.0001)$. Another meta-analysis with meta-regression [37] concluded that GLP-1 RAs and SGLT2is reduce the risk of cardiovascular and mortality endpoints regardless of the reductions in systolic blood pressure and body weight. In patients with T2D with renal impairment, previous research showed that dapagliflozin $10 \mathrm{mg}$ improved weight and systolic pressure, while the benefit on glycemic control was small [38]. When published, weight change results from the DAPA-CKD trial [39] will probably enable further correlations between weight loss and renal outcomes.

The CV advantages obtained with SGLT2 inhibitors in large CVOTs have been only partially clarified by their mechanism of action and related glycemic control. Different approaches have been taken to explain cardiac and renal improvements, which place this class on a different treatment path than the rest of the oral antihyperglycemic agents [4].

\section{DISCUSSION}

\section{Potential Protective Mechanisms of SGLT2 Inhibition Associated with Weight Loss}

Intensive lifestyle measures may result in clinically significant weight reduction (at least 5\%) that can be maintained in the long term [40], and a minimum $3 \%$ reduction in weight is advantageous for metabolic control [41]. In reallife practice, rebound weight gain is common.

The initial decrease in body weight is the result of two major effects of SGLT2 inhibition: caloric loss due to glucose excretion (a process also called calorie restriction mimicry) and loss of body water due to osmotic diuresis. Both mechanisms appear to become effective early after treatment initiation, with glycosuria continuing in the long term without any significant fluid change. We are confident that upcoming studies will clarify whether the weight loss effect is a key component or only an add-on to the impressive cardiorenal outcomes.

The fat loss associated with SGLT2 inhibition is the result of a complex metabolic process described by Ferrannini et al. $[16,42]$ as a gradual shift to fatty acid utilization. The increase in glucagon concentration in response to reduced blood glucose and insulin levels triggers a cascade of metabolic events leading to lipolysis and lipid oxidation. Increased production of ketone bodies, a more efficient source of energy for the heart that require less oxygen for their metabolism, would allow more efficient cardiac contractility, thereby improving the benefits on heart failure. This explanation is offset by the fact that patients with heart failure present high ketone body levels, even in the absence of SGLT2 inhibition. Moreover, ketoacidosis is a trigger of inflammatory conditions, whereas the use of SGLT2is has been proven to reduce inflammation.

Considering the improvement of cardiac function and the associated body fat reduction, the effect of SGLT2 inhibition on epicardial adipose tissue (EAT) was investigated. A small randomized controlled trial $(N=40)$ showed that 6-month dapagliflozin treatment was associated with a significant reduction in EAT volume compared to baseline, with no changes in the control group [43]. This observation is important since arterial stiffness has been correlated with diabetes, obesity, and hypertension. Despite the limited available information, it is likely that SGLT2 inhibitors act by modulating the risk factors, giving them a synergistic larger effect than initially estimated.

\section{SGLT2 Inhibitors, Obesity, and Inflammation}

Obesity is linked to a chronic inflammatory response, with abnormal cytokine and chemokine production. Associated insulin signaling pathways include mitogen-activated protein

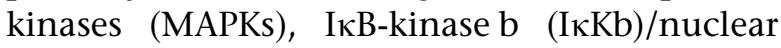
factor $\kappa \mathrm{B}(\mathrm{NF}-\kappa \mathrm{B})$, and mammalian target of rapamycin (mTOR)/S6 kinase [44]. SGLT2 inhibition leads to activation of lysosomal degradation and inhibition of mTOR, with possible 
changes in mitochondrial status followed by a "switch" in inflammatory events. The idea of improving the response to a toxic environment through profound changes in "cellular life history programs" has recently been advanced [45]. This would include the induction of a fastinglike state [46] by SGLT2 inhibition. Such ideas encourage scientists to accept how major improvements are the result of subtle changes.

\section{A Possible Change in Patients' Perspective}

Current guidelines in diabetes are very clear about placing the patient at the center of disease management. Lifestyle changes, including dietary restrictions and an increase in physical activity, form the basis of the treatment and should be adapted to individual needs, and patient preferences should be considered when discussing the overall treatment approach. Metformin remains the first-line pharmacological therapy owing to its effectiveness in both monotherapy and combination therapy, and on the basis of extensive experience [4]. With a neutral overall effect on weight, metformin does not provide cardiorenal protection in patients with diabetes. The paradigm shift from glycemic control to cardiorenal risk control in diabetes places SGLT2is high on the list of pharmacologic agents to be used early in treatment. Additionally, SGLT2is can be used in various therapeutic associations considering their overall efficacy and safety profile and the unique insulin-independent mechanism of action. In clinical practice, the time of intensification with an SGLT2i does not seem to influence the proportion of weight lost [47], but a change of $3-4 \%$ is still lower than anticipated on the basis of the calorie excretion mechanism.

With the development of new and more efficacious treatments for diabetes, the choice of antihyperglycemic agent has become important. In patients with high $\mathrm{CV}$ risk requiring additional disease control, the association of an SGLT2i with GLP-1 RA seems a rational choice. The DURATION 8 study [48, 49] showed that dapagliflozin plus exenatide once weekly improved glycemic control and resulted in a larger weight loss than either drug alone.
Similar results were seen in more recent trials, AWARD-10 [50] and SUSTAIN-9 [51], with other GLP-1 RAs added to a background treatment with an SGLT2i. On the basis of the overall evidence, it seems that sequential or concomitant use of SGLT2is and GLP-1 RA provides the most favorable approach based on their $\mathrm{CV}$ protection (heart failure and major adverse cardiovascular events), glycemic control (HbA1c lowering), and metabolic effects (attenuation of compensatory hyperphagia, weight loss, and minimal risk of hypoglycemia).

For patients and medical staff, T2D management requires an ongoing effort to control the disease. We strongly believe that novel agents associated with weight loss-SGLT2is and GLP-1 RAs-should be exploited at a higher rate. Updated guidelines and current treatment recommendations are a solid basis for this opinion. Although many elements of SGLT2is' potential are still not elucidated, it is likely that physicians go through a clear decision-making process when prescribing them in daily clinical practice.

Interviews conducted with patients with T2D under treatment with one SGLT2i provide a new perspective on patient-reported outcomes [52]. Such qualitative research may represent the starting point for developing a different communication framework with patients who are adherent to SGLT2is that will allow them to pursue other positive behaviors to control diabetes. In the overall perception of their health status, patients with T2D ascribe a more important role to observed weight changes [53] than to HbA1c and blood pressure control. From this point of view, the weight loss effect of SGLT2is, although considered modest and below the clinical significance of $5 \%$ overall, has the potential to become the anchor for our efforts to shift the perspective of patients toward healthy behaviors and adherence to treatment. The structured clinical approach suggested in Fig. 1 has the potential to address both obesity and T2D, aiming to lower the BMI category and promote the cardiorenal benefits of novel therapies.

This short narrative has focused on weight loss attributed to SGLT2is in CVOTs with exclusive T2D populations. The limitations of a 
1. Cardiovascular and renal risk assessment

2. Need for weight loss

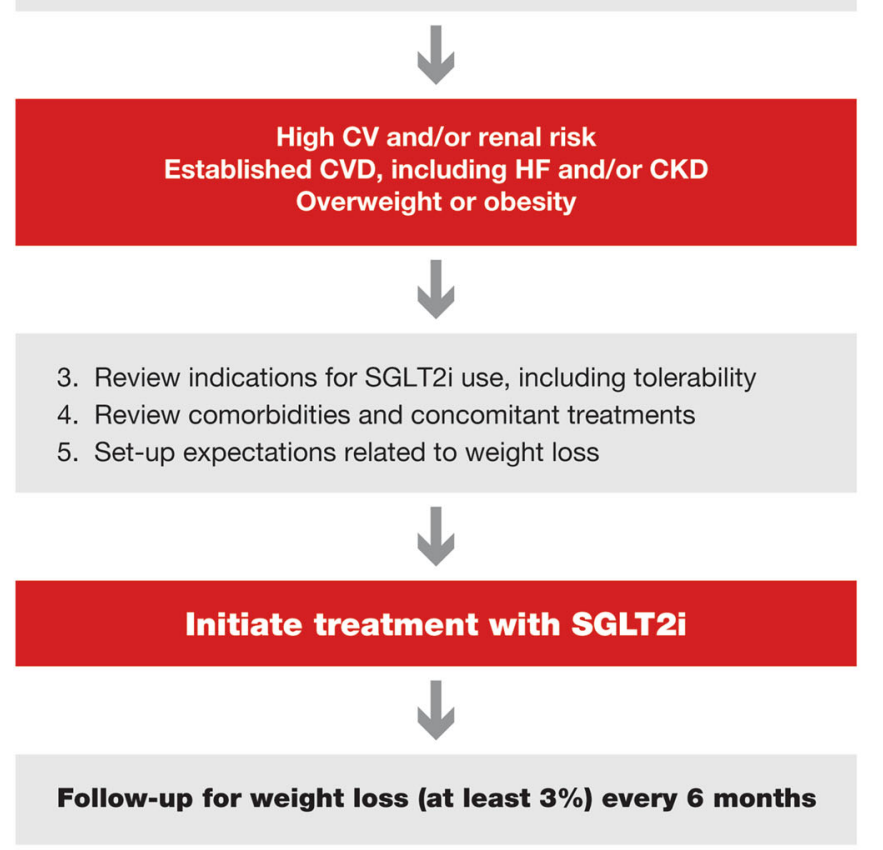

If not achieving desired weight loss

- Consider intensive lifestyle changes and/or association with GLP-1 RA and/or obesity treatment

- Discuss with the patient about the potential risks associated with SGLT2 inhibitors and combination therapies, according to institutional available protocols

CKD, chronic kidney disease; CV, cardiovascular; GLP-1 RA, glucagon-like peptide-1 receptor agonists, HF, heart failure; T2D, type 2 diabetes; SGLT2i, sodium-glucose co-transporter 2 inhibitor.

Fig. 1 Suggested practical algorithm to monitor weight loss in patients with T2D treated with an SGLT2i

brief narrative review are inherent. Details on the chemical and molecular pathways influencing the cardiorenal effects are only marginally introduced. An important topic, such as the effect of SGLT2 inhibition on body weight in subjects without diabetes, is left unexplored. We also did not review in detail the relationship between body weight and renal function in diabetes. Results from clinical practice from a combination of an SGLT2i with GLP-1 RA are not included. The accumulating body of evidence highlights the need to explore weight reduction with SGLT2is in more depth in trials with pragmatic designs to provide more valuable insights that will support individualized treatment approaches.

Despite extensive research, the full implications of weight loss associated with SGLT2 inhibition are largely unknown. The cascade of events triggered by glucose excretion seems very complex, interlacing with inflammation, lipolysis, and deeper intracellular changes, so it might take a long time to solve it completely. The effect on abdominal fat is clearly linked to the obesity reduction, and the increased lipolysis and ketone body formation are linked to the improvement in cardiac metabolism. Future studies and assessments could bring us closer to the clinical predictors of better responses to SGLT2is, with a greater metabolic benefit and BMI improvement.

\section{CONCLUSION}

The mechanisms by which SGLT2 inhibition leads to cardiorenal risk reduction are not fully elucidated but include improved glycemic control, reduced body weight, lower blood pressure, and osmotic diuresis. CV benefits and weight loss are consistently reported with SGLT2is across different patient population groups, irrespective of baseline BMI, moderate chronic disease, and glycemic control. 
Weight reduction remains a crucial goal in the prevention and treatment of diabetes. Along with clinical trials, large real-world studies would allow a better understanding of the appetite patterns and caloric behavior of patients with T2D treated with an SGLT2i to allow this versatile therapeutic class to reach its full potential.

\section{ACKNOWLEDGEMENTS}

Funding. This publication was supported by AstraZeneca UK Limited, Branch Office in Slovenia, Ljubljana, Slovenia (AstraZeneca Slovenia). The Rapid Service Fee was funded by AstraZeneca Slovenia.

Authorship. All named authors meet the International Committee of Medical Journal Editors (ICMJE) criteria for authorship for this article, take responsibility for the integrity of the work as a whole, and have given their approval for this version to be published.

Authors' Contributions. The authors had full editorial control of the manuscript, contributed equally to its development, reviewed and edited various drafts, and provided their final approval of all content.

Medical Writing, Editorial, and Other Assistance. Medical writing assistance was provided by Raluca Voicu, MD of MedInteractiv Plus (Bucharest, Romania) and was funded by AstraZeneca Slovenia.

Disclosures. The authors declare that the current research was conducted in the absence of any commercial or financial relationships that could be construed as a potential conflict of interest. Andrej Janež has served as a consultant and is on speakers' bureaus for AstraZeneca, Boehringer Ingelheim, Eli Lilly, MSD, Novo Nordisk, and Sanofi. Paola Fioretto received personal fees for advisory boards or scientific presentations from AstraZeneca, Mundipharma, Boehringer-Ingelheim, Eli Lilly \& Co, and Novartis.
Compliance with Ethics Guidelines. This article is based on previously conducted studies and does not contain any new studies with human participants or animals performed by any of the authors.

Data Availability. Data sharing is not applicable to this article, as no datasets were generated or analyzed during the current study.

Open Access. This article is licensed under a Creative Commons Attribution-NonCommercial 4.0 International License, which permits any non-commercial use, sharing, adaptation, distribution and reproduction in any medium or format, as long as you give appropriate credit to the original author(s) and the source, provide a link to the Creative Commons licence, and indicate if changes were made. The images or other third party material in this article are included in the article's Creative Commons licence, unless indicated otherwise in a credit line to the material. If material is not included in the article's Creative Commons licence and your intended use is not permitted by statutory regulation or exceeds the permitted use, you will need to obtain permission directly from the copyright holder. To view a copy of this licence, visit http:// creativecommons.org/licenses/by-nc/4.0/.

\section{REFERENCES}

1. Altobelli E, Angeletti PM, Profeta VF, Petrocelli R. Lifestyle risk factors for type 2 diabetes mellitus and national diabetes care systems in European countries. Nutrients. 2020;12:2806.

2. International Diabetes Federation. IDF Diabetes Atlas, 9th Edition. Brussels, Belgium: International Diabetes Federation; 2019

3. Leitner DR, Frühbeck G, Yumuk V, et al. Obesity and type 2 diabetes: two diseases with a need for combined treatment strategies-EASO can lead the way. Obes Facts. 2017;10:483-92.

4. Buse JB, Wexler DJ, Tsapas A, et al. 2019 update to: management of hyperglycemia in type 2 diabetes, 2018. A consensus report by the American Diabetes Association (ADA) and the European Association 
for the Study of Diabetes (EASD). Diabetes Care. 2020;43:487-93.

5. Kanai Y, Lee WS, You G, Brown D, Hediger MA. The human kidney low affinity $\mathrm{Na}+$ /glucose cotransporter SGLT2. Delineation of the major renal reabsorptive mechanism for D-glucose. J Clin Invest. 1994;93:397-404.

6. Wright EM, Loo DD, Hirayama BA. Biology of human sodium glucose transporters. Physiol Rev. 2011;91:733-94.

7. Brown E, Rajeev SP, Cuthbertson DJ, Wilding JPH. A review of the mechanism of action, metabolic profile and haemodynamic effects of sodium-glucose co-transporter-2 inhibitors. Diabetes Obes Metab. 2019;21:9-18.

8. Rajeev SP, Cuthbertson DJ, Wilding JP. Energy balance and metabolic changes with sodium-glucose co-transporter 2 inhibition. Diabetes Obes Metab. 2016;18:125-34.

9. Abdul-Ghani MA, DeFronzo RA, Norton L. Novel hypothesis to explain why SGLT2 inhibitors inhibit only $30-50 \%$ of filtered glucose load in humans. Diabetes. 2013;62:3324-8.

10. Brown E, Wilding JPH, Barber TM, Alam U, Cuthbertson DJ. Weight loss variability with SGLT2 inhibitors and GLP-1 receptor agonists in type 2 diabetes mellitus and obesity: mechanistic possibilities. Obes Rev. 2019;20:816-28.

11. Hall KD, Sacks G, Chandramohan D, et al. Quantification of the effect of energy imbalance on bodyweight. Lancet. 2011;378:826-37.

12. Jurczak MJ, Lee HY, Birkenfeld AL, et al. SGLT2 deletion improves glucose homeostasis and preserves pancreatic beta-cell function. Diabetes. 2011;60:890-8.

13. Devenny JJ, Godonis HE, Harvey SJ, Rooney S, Cullen MJ, Pelleymounter MA. Weight loss induced by chronic dapagliflozin treatment is attenuated by compensatory hyperphagia in diet-induced obese (DIO) rats. Obesity (Silver Spring). 2012;20: 1645-52.

14. Liang Y, Arakawa K, Ueta K, et al. Effect of canagliflozin on renal threshold for glucose, glycemia, and body weight in normal and diabetic animal models. PLoS ONE. 2012; 7:e30555.

15. Polidori D, Sanghvi A, Seeley RJ, Hall KD. How strongly does appetite counter weight loss? Quantification of the feedback control of human energy intake. Obesity (Silver Spring). 2016;24:2289-95.
16. Ferrannini E, Muscelli E, Frascerra S, et al. Metabolic response to sodium-glucose cotransporter 2 inhibition in type 2 diabetic patients. J Clin Invest. 2014;124:499-508.

17. Rajeev SP, Sprung VS, Roberts C, et al. Compensatory changes in energy balance during dapagliflozin treatment in type 2 diabetes mellitus: a randomised double-blind, placebo-controlled, cross-over trial (ENERGIZE)—study protocol. BMJ Open. 2017;7:e013539.

18. Kosugi R, Nakatani E, Okamoto K, Aoshima S, Arai $\mathrm{H}$, Inoue T. Effects of sodium-glucose cotransporter 2 inhibitor (dapagliflozin) on food intake and plasma fibroblast growth factor 21 levels in type 2 diabetes patients. Endocr J. 2019;66:677-82.

19. Scheen AJ. Pharmacodynamics, efficacy and safety of sodium-glucose co-transporter type 2 (SGLT2) inhibitors for the treatment of type 2 diabetes mellitus. Drugs. 2015;75:33-59.

20. Fioretto P, Giaccari A, Sesti G. Efficacy and safety of dapagliflozin, a sodium glucose cotransporter 2 (SGLT2) inhibitor, in diabetes mellitus. Cardiovasc Diabetol. 2015;14:142.

21. Del Prato S, Nauck M, Durán-Garcia S, et al. Longterm glycaemic response and tolerability of dapagliflozin versus a sulphonylurea as add-on therapy to metformin in patients with type 2 diabetes: 4-year data. Diabetes Obes Metab. 2015;17:581-90.

22. Bolinder J, Ljunggren Ö, Kullberg J, et al. Effects of dapagliflozin on body weight, total fat mass, and regional adipose tissue distribution in patients with type 2 diabetes mellitus with inadequate glycemic control on metformin. J Clin Endocrinol Metab. 2012;97:1020-31.

23. Bolinder J, Ljunggren Ö, Johansson L, et al. Dapagliflozin maintains glycaemic control while reducing weight and body fat mass over 2 years in patients with type 2 diabetes mellitus inadequately controlled on metformin. Diabetes Obes Metab. 2014;16:159-69.

24. Neal B, Perkovic V, Mahaffey KW, et al. Canagliflozin and cardiovascular and renal events in type 2 diabetes. N Engl J Med. 2017;377:644-57.

25. Perkovic V, Jardine MJ, Neal B, et al. Canagliflozin and renal outcomes in type 2 diabetes and nephropathy. N Engl J Med. 2019;380:2295-306.

26. Wiviott SD, Raz I, Bonaca MP, et al. Dapagliflozin and cardiovascular outcomes in type 2 diabetes. N Engl J Med. 2019;380:347-57.

27. Wanner C, Lachin JM, Inzucchi SE, et al. Empagliflozin and clinical outcomes in patients with type 2 
diabetes mellitus, established cardiovascular disease, and chronic kidney disease. Circulation. 2018;137:119-29.

28. Cannon CP, Pratley R, Dagogo-Jack S, et al. Cardiovascular outcomes with ertugliflozin in type 2 diabetes. N Engl J Med. 2020;383:1425-35.

29. Ohkuma T, Van Gaal L, Shaw W, et al. Clinical outcomes with canagliflozin according to baseline body mass index: results from post hoc analyses of the CANVAS program. Diabetes Obes Metab. 2020;22:530-9.

30. Jardine M, Zhou Z, Lambers Heerspink HJ, et al. Kidney, cardiovascular, and safety outcomes of canagliflozin according to baseline albuminuria: a CREDENCE secondary analysis. Clin J Am Soc Nephrol. 2021;16:384-95.

31. Oyama K, Raz I, Cahn A, et al. Effects of dapagliflozin on cardiovascular outcomes across body mass index categories in patients with type 2 diabetes mellitus in the DECLARE TIMI 58 trial. J Am Coll Cardiol. 2020;75:660.

32. Pellicori P, Ofstad AP, Fitchett D, et al. Early benefits of empagliflozin in patients with or without heart failure: findings from EMPA-REG OUTCOME. ESC Heart Fail. 2020;7:3401-7.

33. Gallo S, Charbonnel B, Goldman A, et al. Long-term efficacy and safety of ertugliflozin in patients with type 2 diabetes mellitus inadequately controlled with metformin monotherapy: 104-week VERTIS MET trial. Diabetes Obes Metab. 2019;21:1027-36.

34. Heymsfield SB, Raji A, Gallo S, et al. Efficacy and safety of ertugliflozin in patients with overweight and obesity with type 2 diabetes mellitus. Obesity (Silver Spring). 2020;28:724-32.

35. Lopaschuk GD, Verma S. Mechanisms of cardiovascular benefits of sodium glucose co-transporter 2 (SGLT2) inhibitors: a state-of-the-art review. JACC Basic Transl Sci. 2020;5:632-44.

36. Ghosh-Swaby OR, Goodman SG, Leiter LA, et al. Glucose-lowering drugs or strategies, atherosclerotic cardiovascular events, and heart failure in people with or at risk of type 2 diabetes: an updated systematic review and meta-analysis of randomised cardiovascular outcome trials. Lancet Diabetes Endocrinol. 2020;8:418-35.

37. Qiu M, Ding LL, Zhang M, Lin JH, Wei XB, Huang H. GLP-1RAs and SGLT2is reduce cardiovascular events independent of reductions of systolic blood pressure and body weight: a meta-analysis with meta-regression. Diabetes Ther. 2020;11:2429-40.
38. Kohan DE, Fioretto P, Tang W, List JF. Long-term study of patients with type 2 diabetes and moderate renal impairment shows that dapagliflozin reduces weight and blood pressure but does not improve glycemic control. Kidney Int. 2014;85:962-71.

39. Heerspink HJL, Stefánsson BV, Correa-Rotter R, et al. Dapagliflozin in patients with chronic kidney disease. N Engl J Med. 2020;383:1436-46.

40. Wing RR, Bolin P, Brancati FL, et al. Cardiovascular effects of intensive lifestyle intervention in type 2 diabetes. N Engl J Med. 2013;369:145-54.

41. Williamson DA, Bray GA, Ryan DH. Is 5\% weight loss a satisfactory criterion to define clinically significant weight loss? Obesity (Silver Spring). 2015;23:2319-20.

42. Ferrannini E, Baldi S, Frascerra S, et al. Shift to fatty substrate utilization in response to sodium-glucose cotransporter 2 inhibition in subjects without diabetes and patients with type 2 diabetes. Diabetes. 2016;65:1190-5.

43. Sato T, Aizawa Y, Yuasa S, et al. The effect of dapagliflozin treatment on epicardial adipose tissue volume. Cardiovasc Diabetol. 2018;17:6.

44. $\mathrm{Xu} \mathrm{L}$, Ota T. Emerging roles of SGLT2 inhibitors in obesity and insulin resistance: focus on fat browning and macrophage polarization. Adipocyte. 2018;7:121-8.

45. Avogaro A, Fadini GP, Del Prato S. Reinterpreting cardiorenal protection of renal sodium-glucose cotransporter 2 inhibitors via cellular life history programming. Diabetes Care. 2020;43:501-7.

46. Packer M. SGLT2 inhibitors produce cardiorenal benefits by promoting adaptive cellular reprogramming to induce a state of fasting mimicry: a paradigm shift in understanding their mechanism of action. Diabetes Care. 2020;43:508-11.

47. Wilding JPH, Rigney U, Blak BT, Nolan ST, Fenici P, Medina J. Glycaemic, weight, and blood pressure changes associated with early versus later treatment intensification with dapagliflozin in United Kingdom primary care patients with type 2 diabetes mellitus. Diabetes Res Clin Pract. 2019;155:107791.

48. Frías JP, Guja C, Hardy E, et al. Exenatide once weekly plus dapagliflozin once daily versus exenatide or dapagliflozin alone in patients with type 2 diabetes inadequately controlled with metformin monotherapy (DURATION-8): a 28 week, multicentre, double-blind, phase 3 , randomised controlled trial. Lancet Diabetes Endocrinol. 2016;4:1004-16.

49. Jabbour SA, Frías JP, Guja C, Hardy E, Ahmed A, Öhman P. Effects of exenatide once weekly plus 
dapagliflozin, exenatide once weekly, or dapagliflozin, added to metformin monotherapy, on body weight, systolic blood pressure, and triglycerides in patients with type 2 diabetes in the DURATION-8 study. Diabetes Obes Metab. 2018;20:1515-9.

50. Ludvik B, Frías JP, Tinahones FJ, et al. Dulaglutide as add-on therapy to SGLT2 inhibitors in patients with inadequately controlled type 2 diabetes (AWARD-10): a 24-week, randomised, double-blind, placebo-controlled trial. Lancet Diabetes Endocrinol. 2018;6:370-81.

51. Zinman B, Bhosekar V, Busch R, et al. Semaglutide once weekly as add-on to SGLT-2 inhibitor therapy in type 2 diabetes (SUSTAIN 9): a randomised, placebo-controlled trial. Lancet Diabetes Endocrinol. 2019;7:356-67.

52. Huynh J, Menezes HT, Gerstein H, Sherifali D. Exploring the experiences of adults with type 2 diabetes on sodium glucose cotransporter 2 inhibitors. Can J Diabetes. 2020;44:184-91.

53. Woo V, Bell A, Clement M, et al. CANadian CAnagliflozin REgistry: patient-reported outcomes of canagliflozin in the treatment of type 2 diabetes mellitus in Canadian clinical practice. Can J Diabetes. 2019;43:464-71. 